\title{
Network Operation and Management System for Korean fir Ecosystem Monitoring Using Wireless Sensor Network
}

\author{
Kyeseon LEE, Jae Hong RYU \& Nae-Soo KIM \\ ETRI, Daejeon, Korea
}

\begin{abstract}
The existing individual and intermittently, various studies on real-time remote monitoring and permanent fusion of ICT skills in the field of ecological monitoring manual forms have been tried. In this paper, we develop a network operation and management system for Korean fir ecosystem monitoring to collect information on the habitat surrounding environment, including soil moisture, tree diameter growth of information, based on wireless sensor network technology. We present the implementation of the sensor network topology management, sensors, and the node status monitoring / control, sensing data storage and visualization system for performing functions such as for monitoring and managing the system from a remote efficiently.
\end{abstract}

KEYWORD: Monitoring system; Ecosystem; WSN; Network operation and management system

\section{INTRODUCTION}

Recent interest in the ecosystem and the environmental pollution are increasing. In this regard, research and development for ecosystem monitoring techniques for observing ecological changes are being driven by IoT convergence technology [1][6].

Figure 1 represents various ecological information collections.

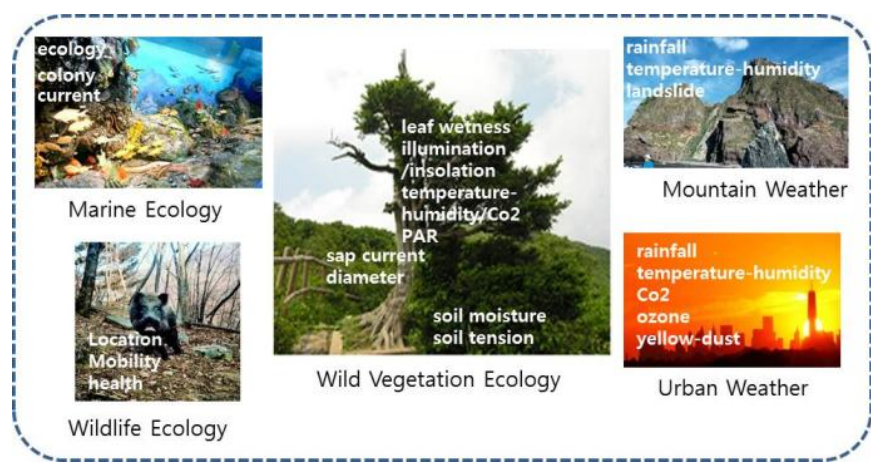

Figure1. Various ecological information collections [2].

Chapter 2 of this paper, we introduce the configuration of the network operation and management information systems for ecological monitoring of wild vegetation communities, and explain how the sensing information transmission over a wireless sensor network. We describe a network management system through UI (User Interfaces) to visualize it after monitoring and constructing databases ecosystem information. We conclude in chapter 3 .

\section{NETWORK OPERATION AND MANAGEMENT SYSTEM FOR KOREAN FIR ECOSYSTEM MONITORING}

\subsection{Overview}

Wild vegetation community's ecological information system configuration of the network operation and management systems is shown in Figure 2.
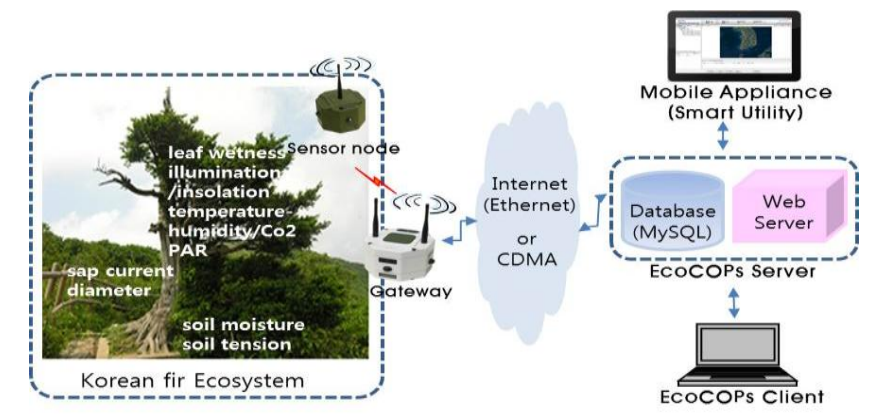

Figure2. Server system diagram [3].

The wireless sensor node is connected a sensor for sensing the sensors to sense the environment information and a tree growing tree information [5]. Sensing information collected in the sensor node is being collected by the gateway, the gateway via a wireless communication network / internet connection is established to the $\mathrm{DB}$ of the network 
operation management system of a real-time monitoring. The system to show the tree growth environment information and information of the tree based on the information of the DB build as a graph, and report the environmental changes over time.

Sensor node acquires sensing data by the interface a variety of sensors, processes the data to have the transmission function to the remote radio [7].

Figure 3 is a testbed site we have installed the sensor nodes and a gateway. Figure 4 shows the wireless performance test conducted in Halla mountain in Jeju Island, target site.

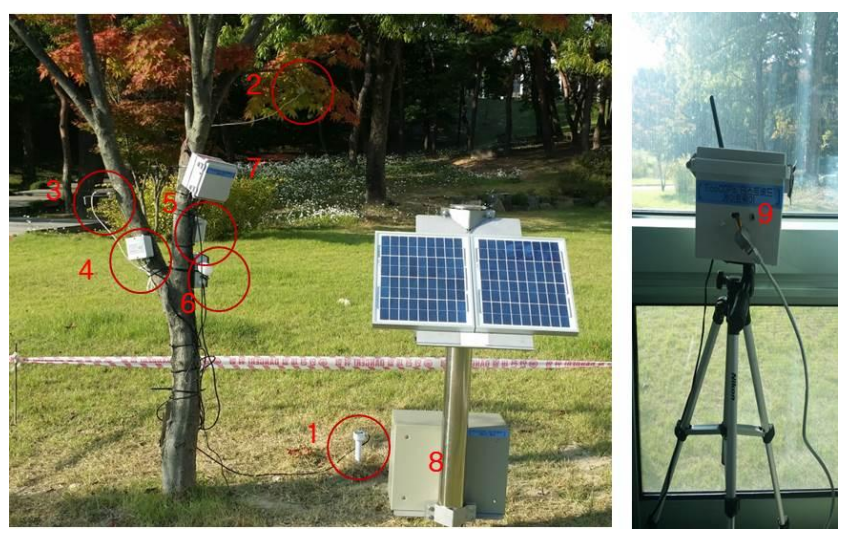

Figure 3. Testbed: Daejeon ETRI 1.Soil moisture 2.leaf wetness 3.diameter 4.temperature-humidity 5.Illumination /insolation 6.Co2 7.Sensor node 8.Battery module 9.Gateway: in-door
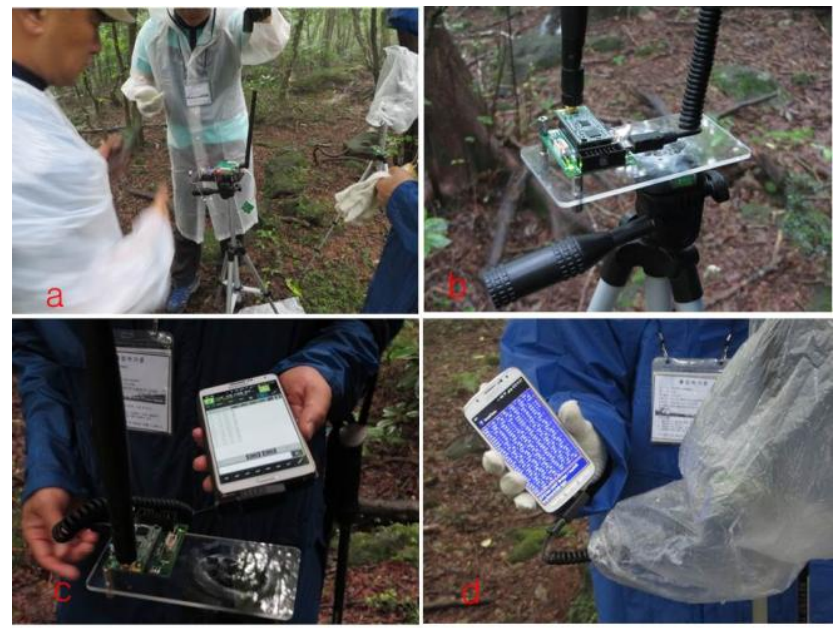

Figure4. Testbed: Halla mountain in Jeju Island (a) raining day, $925 \mathrm{MHz}$ band, over 200M (b) Sending node (c)(d) receiving node: check RSSI of receiving packets

\subsection{System construction}

The system collects data and create a database after connect to the gateway Using Ethernet or CDMA. It illustrates the information visible by the HMI based on the database. It provides the ability to share the stored database and ecological prediction system. The system runs on regular PC and uses Windows as the default operating system and provides UI. It provides a smart phone-based client application App. There is also access to move the manager, providing Android as the default OS[4].
Table 1. System Preferences.

\begin{tabular}{|c|l|}
\hline & \multicolumn{1}{|c|}{ Features } \\
\hline $\begin{array}{c}\text { Operation } \\
\text { Evironment }\end{array}$ & - PC \\
\hline $\begin{array}{c}\text { Screen indication } \\
\text { functions }\end{array}$ & $\begin{array}{l}\text { - Sensort Utility: Phone, Pad, Tap } \\
\text { - Available in graph form }\end{array}$ \\
\hline External & - Interface with wired/wireless network \\
Interface & - Interface with Sensing information \\
& Analysis/Prediction System \\
\hline & - GUI(Graphic User Interface) \\
& - Network Management \\
Main & - User Input Management \\
- Operation Mode Management \\
- CCTV Management \\
& - Event Data Management \\
& - Alarm settings, and disable function \\
\hline
\end{tabular}

Detailed functional configuration is shown in Figure 5 below. In this section, we describe a detailed description of each function-specific.

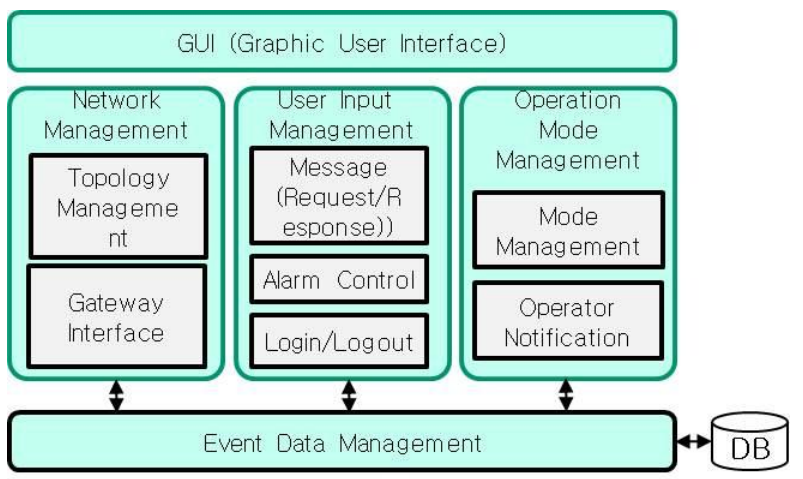

Figure5. Functional Structure of Network Operation and Management System

- GUI: This topology wild vegetation cover of the sensor network configuration image as wallpaper, located in the center of figure6. For information about the network and event data received and sent messages are written to the message log window. On the left screen, an operation mode window and an instruction window to control nodes are located.

- Network Management: The network topology management manages the connection state and configuration information for the sensor network configured. The upper left indicates the network / node management screen. The bottom left indicates the selected sensor node's properties screen.

- Event Data Management: If the event data is generated by receiving the sensor node received risk information displayed on the GUI screen, and shows the alarm information. Alarm information is managed in a database to enable query history by the operator. Figure7 displays graphics for sensing data.

$\checkmark$ Soil moisture: volumetric soil water content 
$\checkmark$ Leaf wetness: measuring the degree of moisture present in the leaves

$\checkmark$ Diameter: measuring the degree of tree growth

$\checkmark$ Temperature-humidity: study the growth of trees and associations

$\checkmark$ Illumination /insolation: study the growth of trees and associations using sensor for measuring the intensity or brightness of the sunlight

$\checkmark$ Co2: study the growth of trees and associations

- User Input Management: It is responsible for the ability to send a user command to the sensor node and may select a node on the screen to send a command to send commands to select or command window. It performs GUI-based management program startup user login / logout functionality and manages the user's ID/PW in figure8.

- Operation Mode Management: The event manager notifies the event to the text message, and displays the operating mode of the system.

$\checkmark$ Off mode: mode to inspect system and operate by administrator

Alarm mode: mode to monitor the battery level and an abnormal detachment of node as figure9.

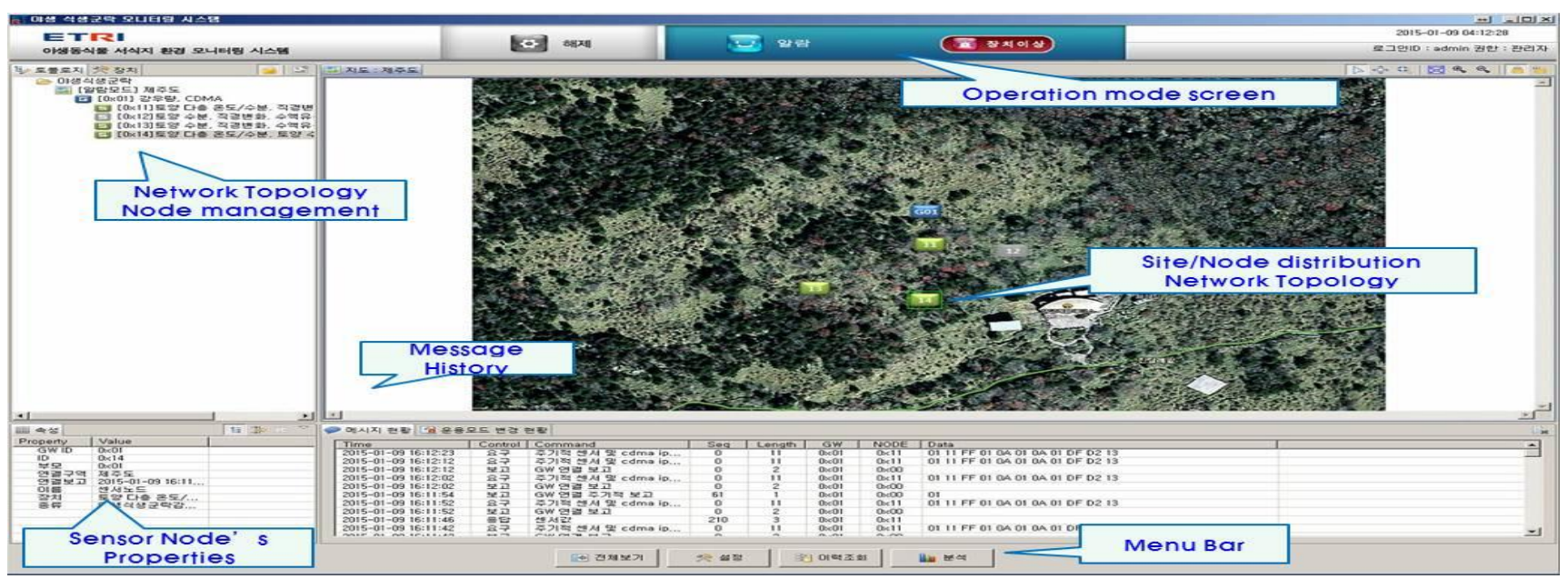

Figure 6. Network Topology Management Screen

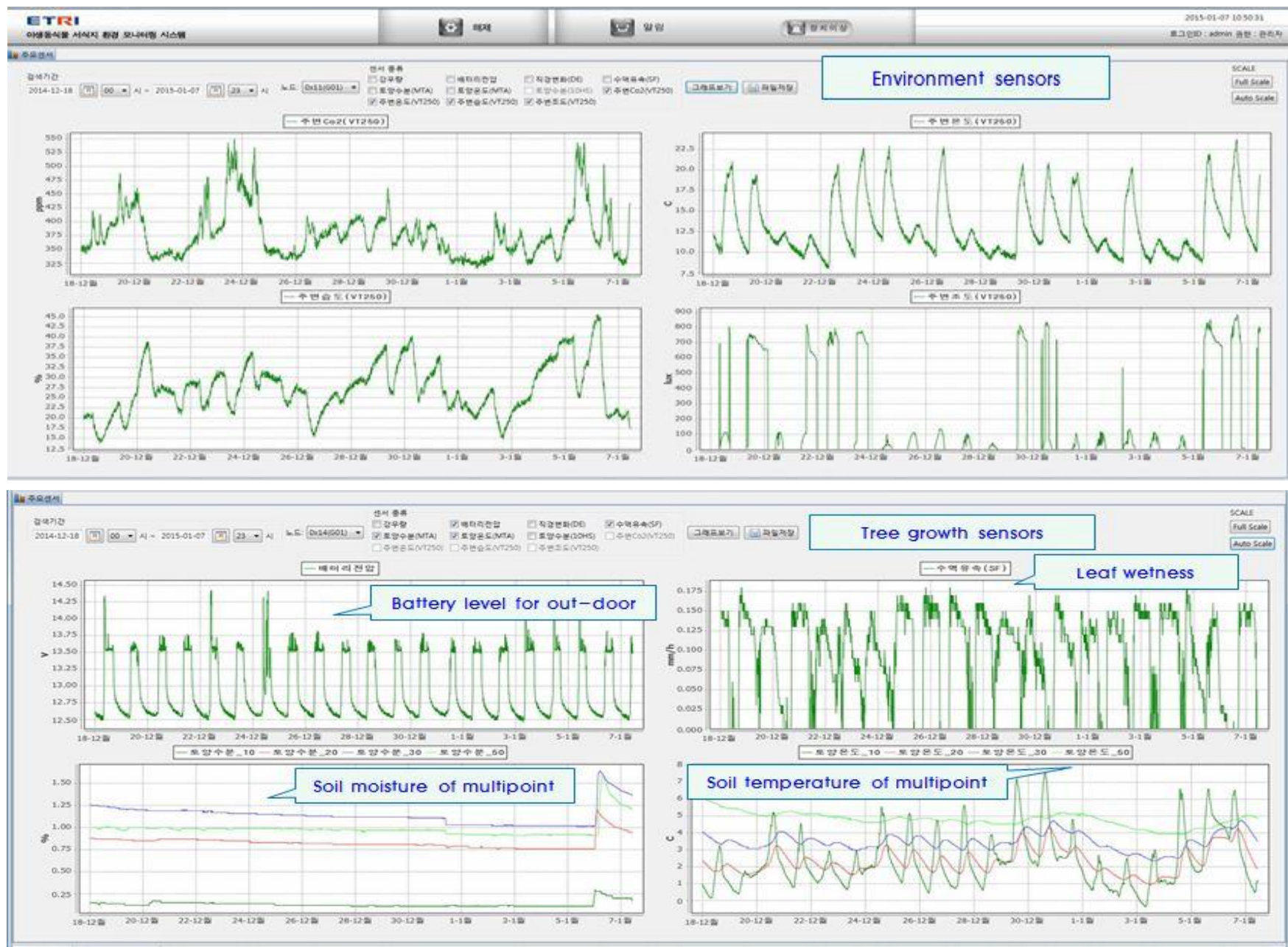


Figure7. Graphics for sensing values $(\uparrow)$ Co2, Temperature, humidity, Illumination $(\downarrow)$ Battery, Leaf-wetness, Soil moisture, soil temperature

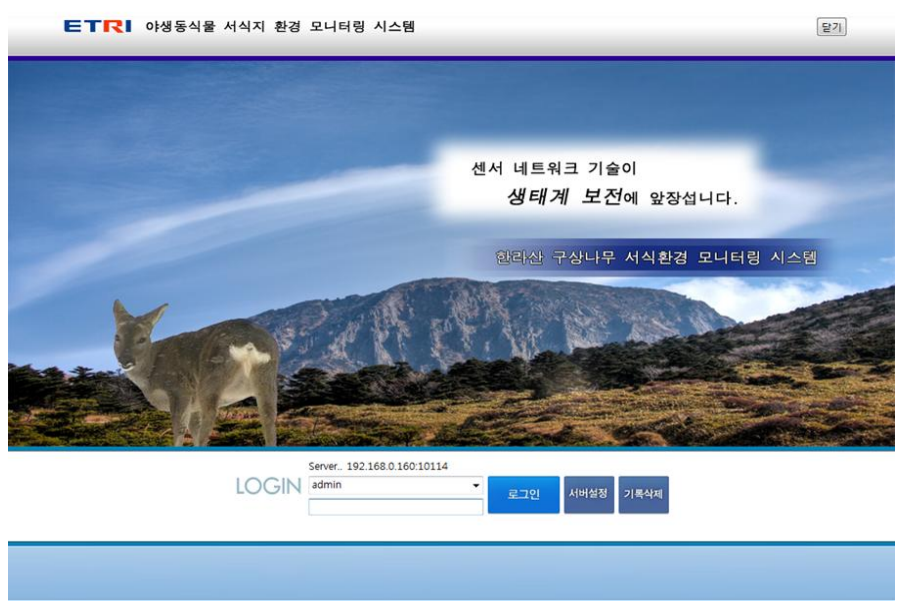

Figure8. Login screen

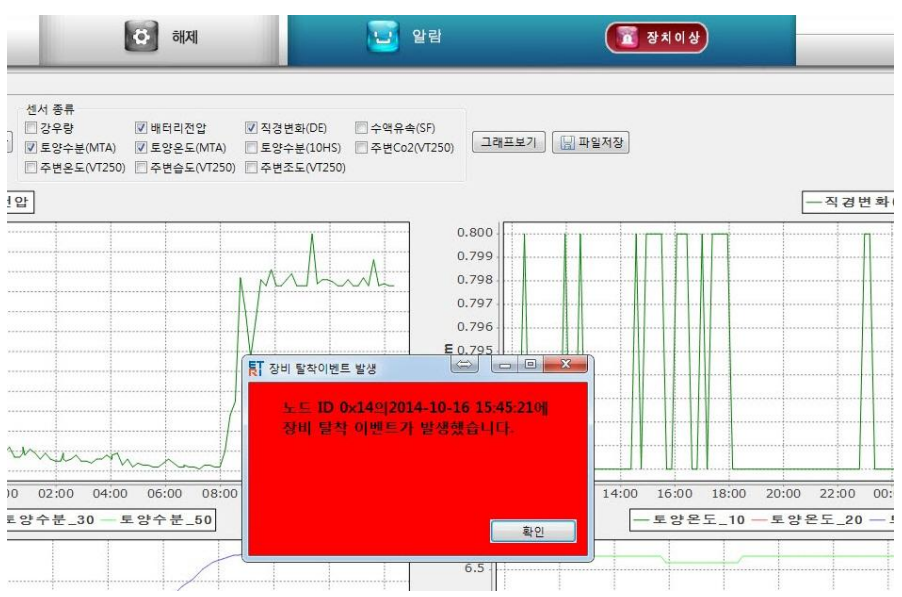

Figure9. Alarm screen: Node's abnormal detachment

\section{CONCLUSIONS}

To select a wild ecosystem to observe, you have to consider the characteristics of the area. Or you may consider a target site, certain plants, certain animals and certain mountain. The system is constructed in this paper proceeds by selecting the Korean fir ecosystem in Halla mountain in Jeju island to the site. In consideration of the characteristics of this site, we must apply low-power sensor networks. The best selection of sensors is required for this purpose.

Korean fir are inhabited only the Republic of Korea and placed on the impact of global warming since 2100 is known to be highly likely extinction. We need to research a certain tree and its environment that is worth preserving. Out research will be associated with ecology, ecological scientists will be provided a variety of information needed.

\section{ACKNOWLEDGMENT}

This work was supported by Ministry of Environment of ROK project number 2013000210002

\section{REFERENCES}

[1] Nae-Soo Kim et al., "Trends of Ecological Changing Monitoring and IoT Convergence Technology," Electronics and Telecommunications Trends, 2013

[2] Nae-Soo Kim et al., "Conceptual Architecture of Low power Sensor Networks for Real-time Ecological Change Monitoring," The 68th conference of the Korean Association of Biological Sciences

[3] Jae-Hong Ryu et al., "Gateway System for Realtime Monitoring Wild Animal and Plant," The conference of Embedded Systems and Applications, 2014

[4] Kyeseon Lee et al., "Implementation of Network Operation and Management System for Wildlife Ecosystem Monitoring Using Wireless Sensor Networ," CICS2014

[5] K. Smettem et al., "Environmental Monitoring with Sensor Networks," University of Western Australia Wireless Sensor Network Group

[6] http://www.klter.org "Korea Long-Term Ecological Reserch (KLTER) Network"

[7] http://www.ti.com/.. 